\title{
XII INTERNATIONAL WORKSHOP ON QUANTUM OPTICS (IWQO-2015) PREFACE AND PRESENTATION
}

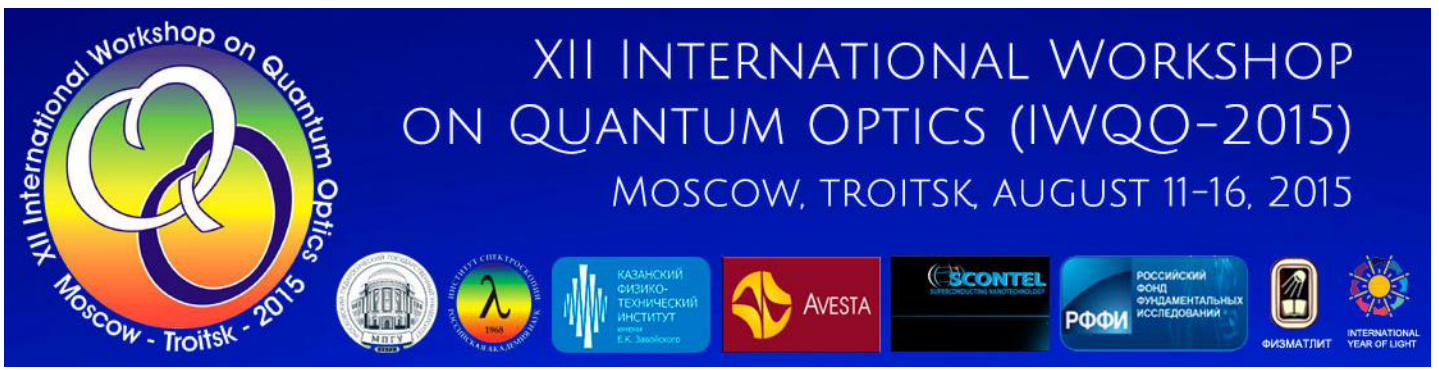

XII International Workshop on Quantum Optics (IWQO-2015) is a regular conference on relevant problems of quantum optics. It is held in Russia with the participation of representatives of foreign research groups. The first workshop was organized in October 1989 in Kazan by Prof. Vitaly Samartsev (Zavoisky Institute of Physics and Technology, Kazan, Russia). Currently the Workshops are held quadrennially in different places of Russia (2003 - St. Petersburg, 2007 - Samara, 2011 Volgograd).

IWQO-2015 was held in Moscow (south-west and Troitsk city districts) on the base of Moscow State Pedagogical University (www.mpgu.edu) and Institute for Spectroscopy of the Russian Academy of Sciences (www.isan.troitsk.ru). The Workshop was included into the list of events of the International Year of Light and Light-based technologies (IYL-2015). Chairmens of the Program Committee: Prof. Dr. Vitaly Samartsev, Head of the Nonlinear Optics Laboratory in ZPhTI RAS and Prof Dr. Evgeny Vinogradov, corresponding member of the Russian Academy of Sciences, Head of the Solid State Spectroscopy Department in the Institute for Spectroscopy RAS. The IWQO-2015 topics cover a broad range of actual questions of quantum optics and related fields, including quantum informatics, perspective quantum communications and simulations, spectroscopy, laser physics, nanooptics, photonics, physics of the nonlinear and ultrafast phenomena, physics and physical chemistry of novel materials, spectral instrumentation.

This issue of the EPJ Web of Conferences consists of the expanded version of abstracts of the selected talks presented at the IWQO-2015. The issue is recommended for specialists who work in the field of quantum optics, as well as for M.S. and Ph.D. students. The full program and details about the Workshop can be found on the web-site www.iwqo2015.ru

The Workshop IWQO-2015 was supported by Russian Foundation for Basic Researches (www.rfbr.ru), "AVESTA" (manufacturing of lasers and optical components, www.avesta.ru) and "SCONTEL" (manufacturing of the single photon detectors, www.scontel.ru) companies, and the publishing house "FIZMATLIT" ( $\underline{\text { www.fml.ru}})$.

Organizing committee head Prof. Dr. A.V. Naumov. Scientific secretary Dr. K.R. Karimullin.

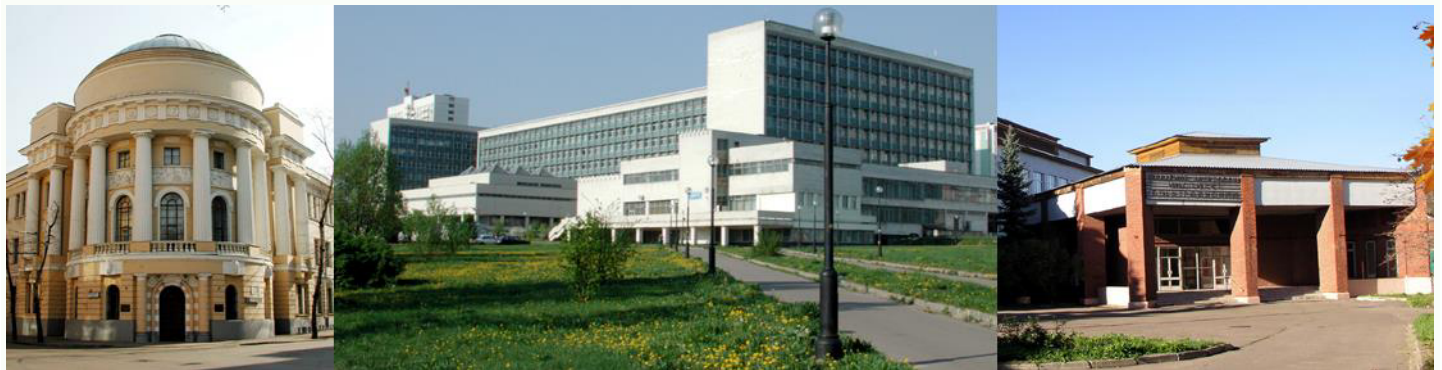

This is an Open Access article distributed under the terms of the Creative Commons Attribution License 4.0, which permits unrestricted use, distribution, and reproduction in any medium, provided the original work is properly cited. 
The Conference program included 122 talks of 284 authors from 13 countries (Russia, Armenia, Canada, Estonia, France, Germany, Kazakhstan, Netherlands, Poland, South Africa, UK, Ukraine, USA).

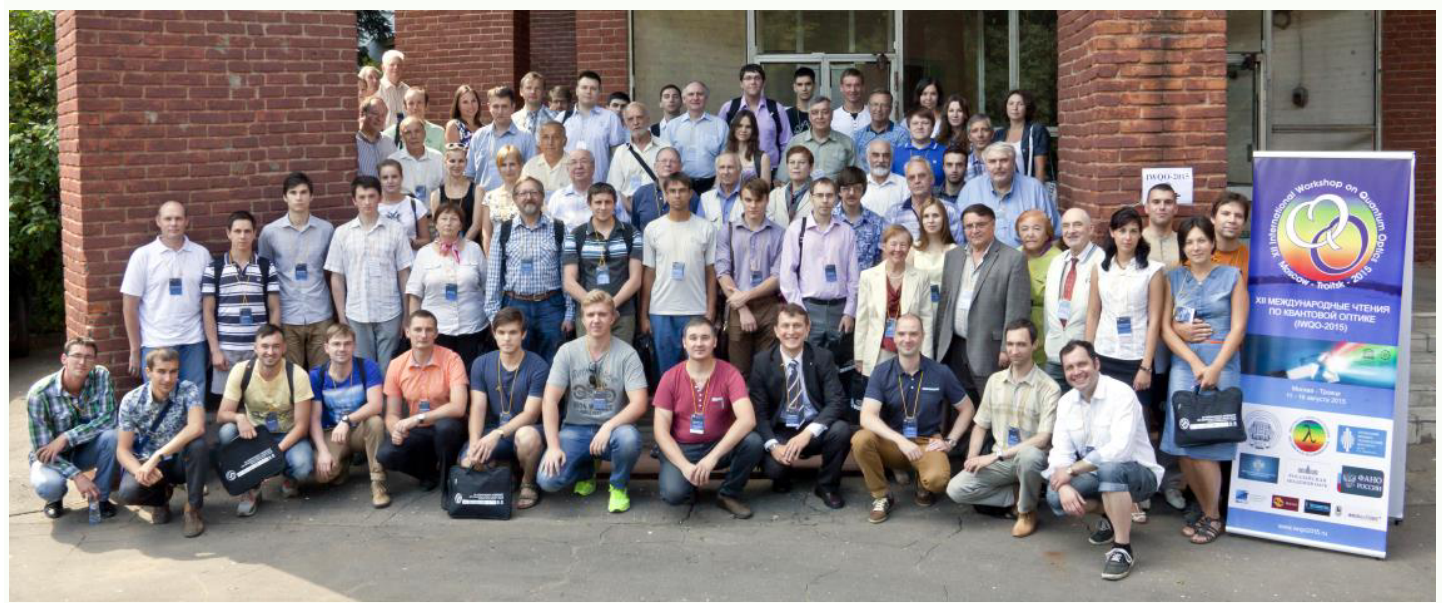

During the Conference a Scientific School of Young Scientists "Actual problems of quantum optics" and the contest for the best scientific paper award were organized. Young authors of the best reports were awarded with valuable prizes and diplomas of the Conference.

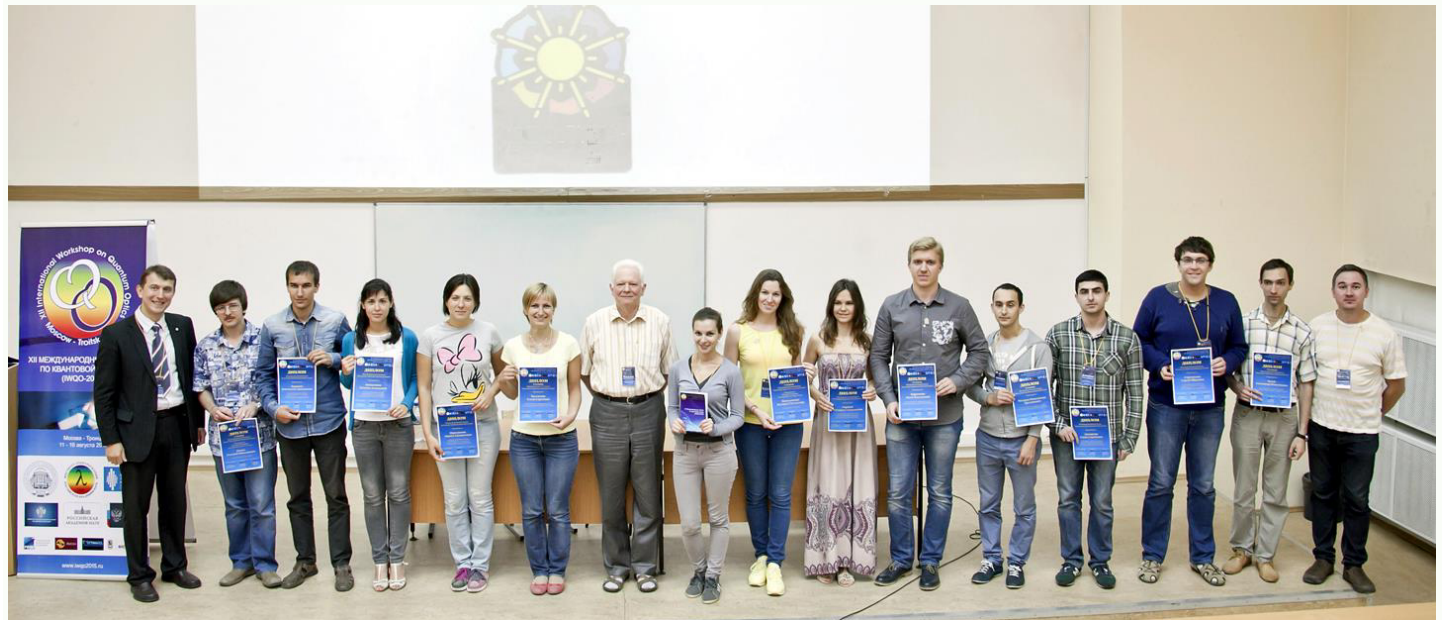

In addition to scientific sections, a cultural program including lab tours, city tours and visits to historical places was held.

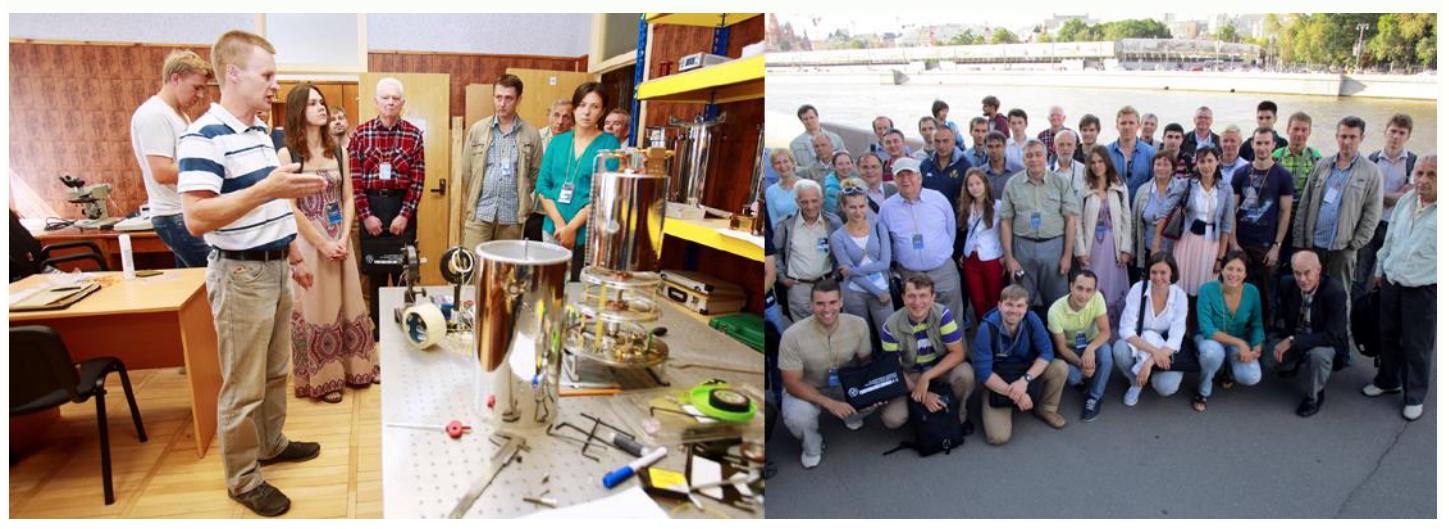

